\title{
Redox PotentialSensor Arrayby Extended-Gate FET with Ferrocenyl-Alkanethiol Modified Gold Electrode
}

\author{
Hiroo Anan ${ }^{1}$, Masao Kamahori ${ }^{2}$, Yu Ishige ${ }^{2}$, Kazuo Nakazato ${ }^{1}$ \\ ${ }^{1}$ Department of Electrical and Computer Science, Graduate School of Engineering, Nagoya University, \\ Furo-cho, Chikusa-ku, Nagoya 464-8603, Japan, \\ h_anan@echo.nuee.nagoya-u.ac.jp \\ ${ }^{2}$ Central Research Laboratory, Hitachi, Ltd., 1-280 Higashi-Koigakubo, Kokubunji, Tokyo 185-8601, \\ Japan
}

\begin{abstract}
:
We developed a $32 \times 32$ array chip of extended-gate FET-based redox potential sensor with 11ferrocenyl-1-undecanethiol (11-FUT) modified gold electrode. The sensor array detected the redox reaction of hexacyanoferrate (II) and hexacyanoferrate (III) as a change in the electric potential of the 11-FUT modified electrode in accordance with the Nernstian response at a slope of $57.9 \mathrm{mV} / \mathrm{decade}$ at $25^{\circ} \mathrm{C}$ with a dynamic range of more than five orders of magnitude. The stability of the potential was within $0.5 \mathrm{mV} / \mathrm{h}$. Of all $32 \times 32$ sensor cells, each potential of $80 \%$ and each difference of $92 \%$ were within $\pm 5 \mathrm{mV}$ and $\pm 1 \mathrm{mV}$ from median, respectively. The 2-dimensional and real time visualization were enabled by imaging of sensor array. Using an enzyme-catalyzed redox reaction, this FET-based sensor array successfully detected a glucose level from 25 to $200 \mathrm{mg} / \mathrm{dL}$.
\end{abstract}

Key words: Extended-gate FET sensor array, Redox potential sensor, Glucose sensor, Ferrocenylalkanethiol, Nernst equation, Chemical reaction imaging

\section{Introduction}

Field-effect transistor (FET)-based biosensors are energetically studied because of their attractive features such as small size, low cost, and the possibility of on-chip integration of multimodal biosensors. Recent studies showed that extended-gate FET sensor could detect charges of biomolecules [1][2]. However, these FET sensors that directly detect biomolecular charges are strongly affected by the buffer conditions such as $\mathrm{pH}$ and salt concentration, which cause decrease in detection efficiency or unstableness of electric potential of the electrode when the salt concentration becomes low in order to avoid screening by ions. Instead of direct charge detection method, redox reaction detection method was developed using an extended-gate FET with 11-FUT modified gold electrode [3]. This redox potential sensor detects the ratio of oxidizer to reducer concentration, and is not affected by the absolute concentrations and $\mathrm{pH}$, thus the sensor can detect biomolecules with high stability and high accuracy. In this paper, we report a chip which integrates $32 \times 32$ redox potential sensors and the controlling circuit.

\begin{abstract}
Fabrication of FET-based sensor array chip
The structure of on-chip $32 \times 32$ extended-gate FET sensor array with 11-FUT modified gold electrodes is shown in Fig. 1. The FET-based sensor array chip was fabricated in a standard $1.2 \mu \mathrm{m} 2$-metal and 2-poly CMOS process(ON Semiconductor). Layers of $20 \mathrm{~nm} \mathrm{Ti}$ and $100 \mathrm{~nm}$ $\mathrm{Au}$ were deposited on the extended-gate electrodes, and patterned by optical lithography and wet etching.SU-8 layer is adopted on the chip to protect from the solution, resulting in a $20 \mu \mathrm{m} \times 56 \mu \mathrm{m}$ opening of electrode. CMOS source-drain follower circuit was used to output the potential of the extended-gate electrode [4].Therefore, the electrode potential can be measured in real time.

The alkanethiol-modification procedure of the gold electrodes of extended-gate FET sensor array was as follows. The chip was kept in an alkanethiol solution for $24 \mathrm{~h}$. The concentration of ferrocenyl-alkanethiol solution (11-FUT) was $500 \mu \mathrm{Min}$ ethanol. After alkanethiol modification, the chip was rinsed with ethanol and deionized water twice to remove unreacted alkanethiol molecules. The chip was kept in a 0.1-M sodium sulfate solution at room temperature until use.
\end{abstract}




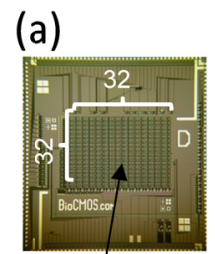

(b) su-8 layer

FET sensor array

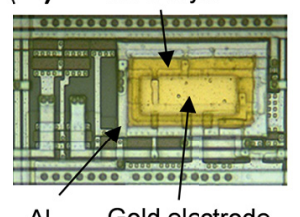

Al Gold electrode

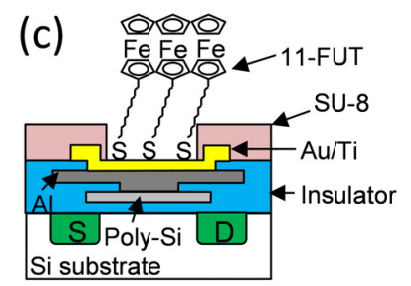

Fig. 1. Optical photograph of (a) sensor array and (b) sensor unit, and (c) structure

\section{Measurement principle of FET-based redox potential sensor}

The change in the ratio of redox compound can be detected by the ferrocenyl-alkanethiol modified-FET sensor as the interfacial potential. In this study, hexacyanoferrate ions were used as the redox compound due to their high reactivity with ferrocene compounds. The scheme of the redox reaction between the hexacyanoferrate ions and the immobilized ferrocene molecules on the gold electrode of an extended-gate FET sensor is shown in Fig. 2.Whenferrocene immobilized on the gold electrode coexists with hexacyanoferrate(III) and hexacyanoferrate (II) in the solution, the redox reaction is given by

$$
\mathrm{Fc}+\left[\mathrm{Fe}(\mathrm{CN})_{6}\right]^{3-} \underset{\leftarrow}{\leftarrow} \mathrm{Fc}^{+}+\left[\mathrm{Fe}(\mathrm{CN})_{6}\right]^{4-}
$$

The relation among the concentrations of ferrocene ([Fc]), ferrocenium ion $\left(\left[\mathrm{Fc}^{+}\right]\right)$, hexacyanoferrate (III) $\left(\left[\left[\mathrm{Fe}(\mathrm{CN})_{6}\right]^{4-}\right]\right)$, and hexacyanoferrate $(\mathrm{II})\left(\left[\left[\mathrm{Fe}(\mathrm{CN})_{6}\right]^{3-}\right]\right)$ is given by eq. (2) using an equilibrium constant $K$,

$$
\left.\left[\mathrm{Fc}^{+}\right]=\mathrm{K}, \frac{\left[\mathrm{Fe}(\mathrm{CN})_{6}\right]^{4-}}{\left[\mathrm{Fe}(\mathrm{CN})_{6}\right]^{3-}}\right]
$$

The interfacial potential $E$ on the ferrocene immobilized gold electrode is derived from the Nernst equation as follows,

$$
E=E_{\mathrm{Fc}}^{0}-\frac{R T}{F} \ln \frac{[\mathrm{Fc}]}{\left[\mathrm{Fc}^{+}\right]}
$$

where $E_{\mathrm{Fc}}^{0}$ is the standard electrode potential on the ferrocene immobilized gold electrode, $R$ is the gas constant, $T$ is absolute temperature, and $F$ is the Faraday constant. By substituting eq. (2), eq. (3) can be rewritten using the concentration of hexacyanoferrate as follows,

$$
\left.E=E^{0}-\frac{R T}{F} \ln \frac{\left[\mathrm{Fe}(\mathrm{CN})_{6}\right]^{4-}}{\left[\mathrm{Fe}(\mathrm{CN})_{6}\right]^{3-}}\right]
$$

where $E^{0}$ is a constant including the standard electrode potential and equilibrium constant.
Eq. (4) shows that the ratio of hexacyanoferrate (II) concentration to hexacyanoferrate (III) concentration can be detected by measuring the interfacial potential of the ferrocene immobilized gold electrode. At $25{ }^{\circ} \mathrm{C}$, the slope of eq. (4) is $59 \mathrm{mV} /$ decade theoretically.

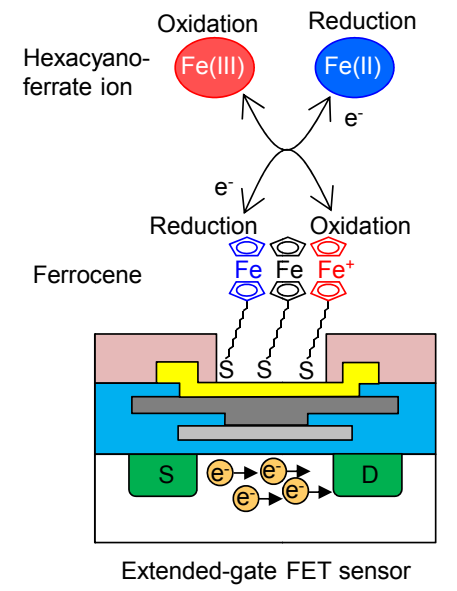

Fig. 2. Redox reaction between the hexacyanoferrate ions and the immobilized ferrocene molecules on the gold electrode.

\section{Measurement Results}

The stability of interfacial potential of the FETbased redox potential sensor array was examined in the following procedure. Two types of FET sensor array chips, non-modified chip and 11-FUT modified chip were used. These sensor array chips and $\mathrm{Ag} / \mathrm{AgCl}$ reference electrode with saturated $\mathrm{KCl}$ were immersed in two types of solutions, P'BS and Redox-PBS (PBS containing total $10 \mathrm{mM}$ hexacyanoferrate (II) and hexacyanoferrate(III). Fig. 3 shows the stability of the interfacial potential. In the case of bare electrode, the potential drift of $34 \mathrm{mV} / \mathrm{h}$ was observed in PBS solution. The drift was reduced to $2 \mathrm{mV} / \mathrm{h}$ in redox PBS solution since the potential was rapidly fixed at the redox equilibrium potential. Furthermore, the stability of the potential was drastically improved, within $0.5 \mathrm{mV} / \mathrm{h}$, by employing 11-FUT modified gold electrode since the electrode is protected from the corrosion by solution.

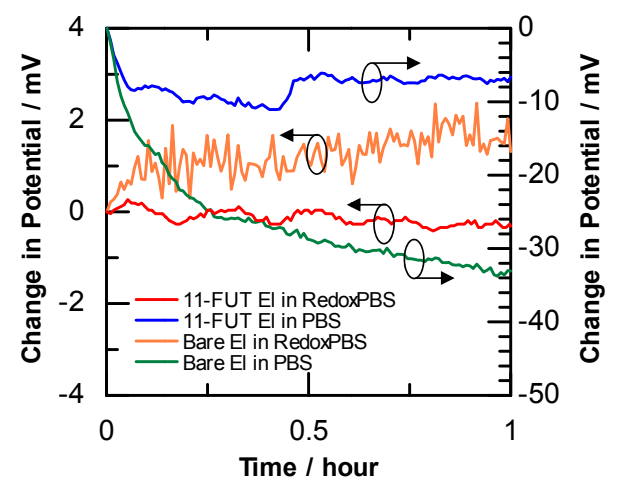

Fig. 3. Time-course change of potential (median) measured using the redox potential sensor array 
The dependency of interfacial potential of the FET-based redox potential sensor array onthe concentration ratio of hexacyanoferrate (II) to hexacyanoferrate(III) was obtained in the following procedure. The 11-FUT modified FET sensor array and the $\mathrm{Ag} / \mathrm{AgCl}$ reference electrode with saturated $\mathrm{KCl}$ were immersed in each of the mixed solutions of hexacyanoferrate(II) and hexacyanoferrate (III) at a ratio of 1:999, 1:99,1:9, 1:1, 9:1, 99:1, and $999: 1$. The total concentration of the mixed solution was $10 \mathrm{mM}$.Fig. 4 shows the relationship between the ratio of hexacyanoferrate (II) to hexacyanoferrate (III) concentration and the interfacial potential measured by using the 11-FUT modified FET sensor array. The interfacial potential showed a fairly good linearity $\left(r^{2}=0.9996\right)$ for a concentration ratio from $10^{-2}$ to $10^{3}$ and the slope was $57.9 \mathrm{mV} /$ decade, almost the same as the theoretical value of the Nernst equation at room temperature.

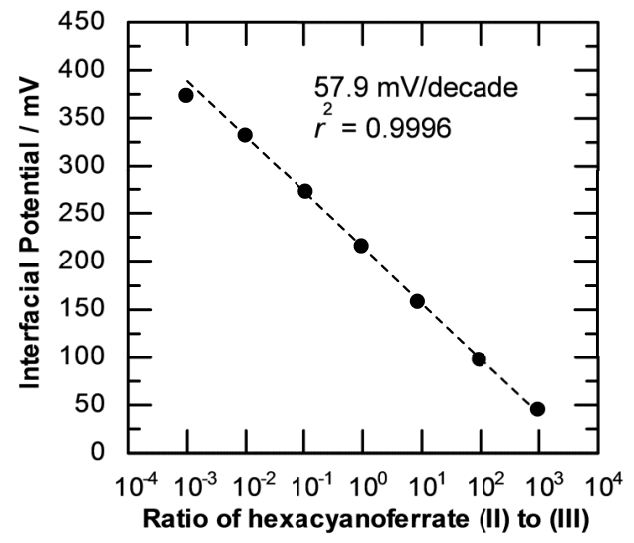

Fig. 4. Interfacial potential (median) versus ratio of hexacyanoferrate (II) to hexacyanoferrate (III) concentration

Fig. 5 (a) and (b) show the cumulative probabilities of potential at each ratio of hexacyanoferrate (II) to hexacyanoferrate (III) concentration and the difference between each ratio and ratio 1 , respectively. Of all $32 \times 32$ sensor cells, each potential of $80 \%$ and each difference of $92 \%$ were within $\pm 5 \mathrm{mV}$ and \pm 1 $\mathrm{mV}$ from median, respectively. The variation \pm 5

(a)

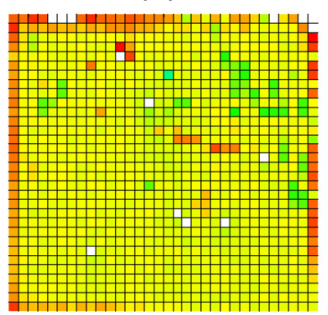

(b)

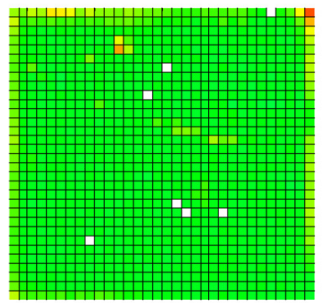

$\mathrm{mV}$ is mainly attributed to the variation of threshold voltage of pair transistors [4], which can be eliminated by taking the difference of two sensed voltages.
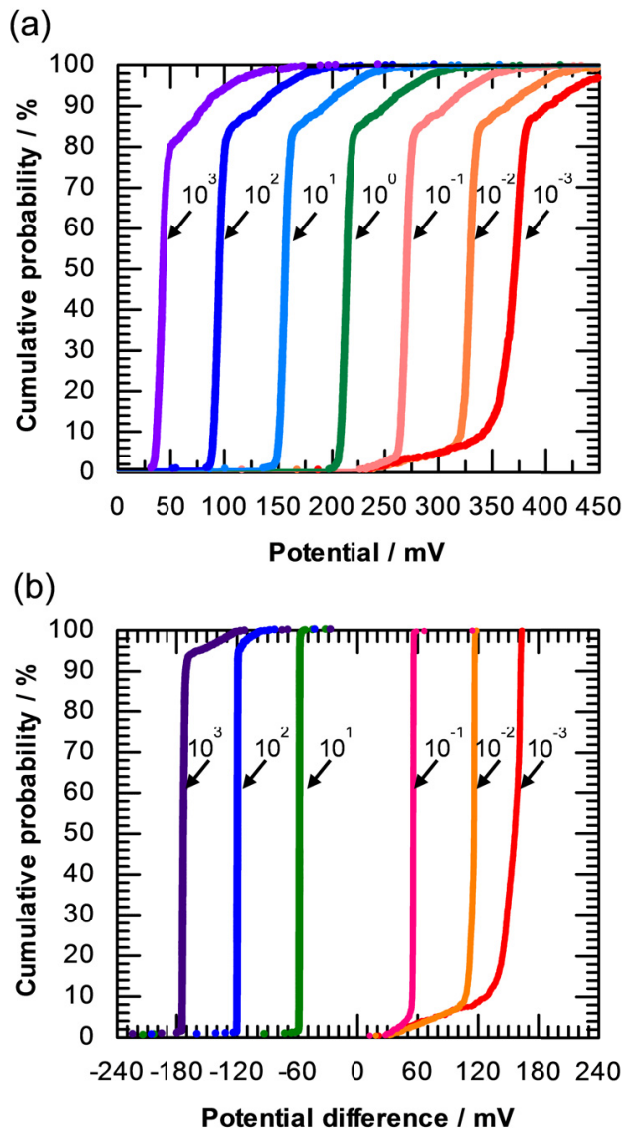

Fig. 5. Cumulative probability of (a) potential at each ratio of hexacyanoferrate (III) to hexacyanoferrate (II) concentration, and (b) the potential difference between each ratio and ratio 1 of hexacyanoferrate (III) to hexacyanoferrate (II) concentration.

Fig. 6 shows imaging of $32 \times 32$ sensor array at each ratio of hexacyanoferrate (II)to hexacyanoferrate (III) concentration. A redox potential image sensor, which can observe twodimensional chemical reactions in real time, was successfully realized.

(c)

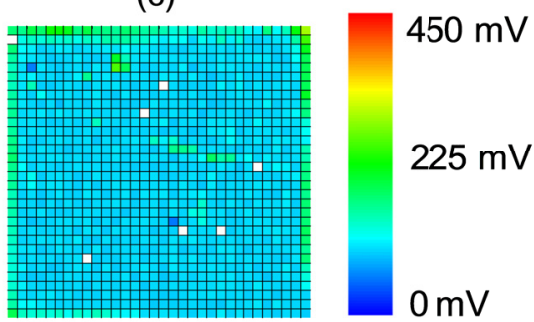

Fig. 6. Image of potential in $32 \times 32$ sensor array at the ratio of hexacyanoferrate (II)to hexacyanoferrate (III) concentrations of (a) $10^{-2}$, (b) $10^{0}$, and (c) $10^{2}$ 
This redox potential sensor array was applied to the detection of glucose. The following enzyme reaction system for glucose was used,

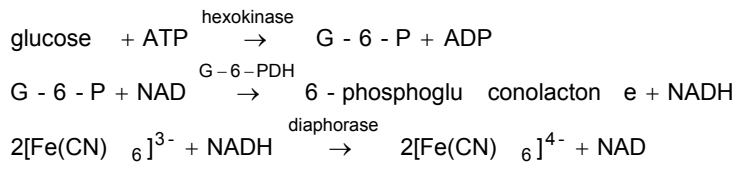

where ATP is adenosine triphosphate, ADP is adenosine diphosphate, NAD is the nicotinamide adenine dinucleotide, $\mathrm{NADH}$ is a reduced form of NAD, G-6-P is glucose-6phosphate, and G-6-PDH is glucose-6phosphate dehydrogenase. The enzyme reaction system converts $1 \mathrm{~mol}$ of glucose to 2 mol of hexacyanoferrate (II), which can be detected by the 11-FUT modified FET sensor as mentioned above. Enzyme-mixed solution (Redox-PBS mixed with Hexokinase, G-6-PDH, Diaphorase, $\mathrm{MgCl}_{2}, \mathrm{NAD}$, and ATP) and

(a)

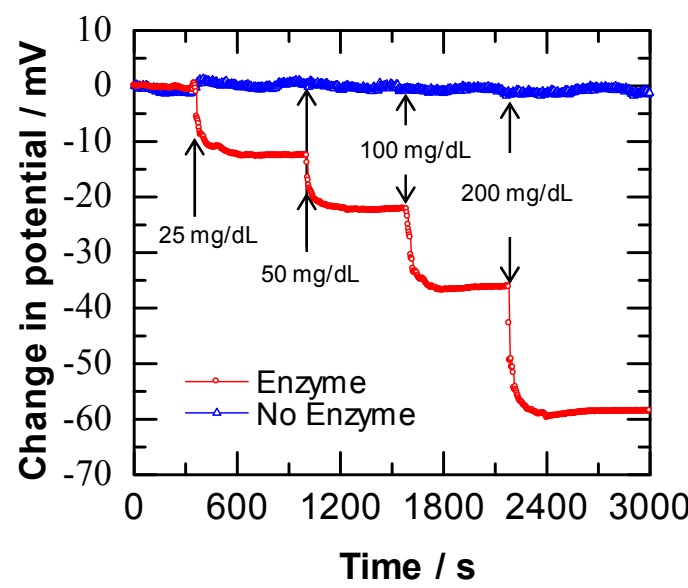

glucose solutions (PBS mixed with glucose, the glucose concentration were $25,50,100$, and $200 \mathrm{mg} / \mathrm{dL}$ ) were mixed with at a ratio of 10:1. The measurement of the potential change of the11-FUT modified FET sensor array was performed in the same way as in the measurement of the mixed solution of hexacyanoferrate (II) and hexacyanoferrate (III). Fig. 7 (a) shows the time-course change in the potential measured for stepwise changes in a glucose level. When the sample of each concentration was supplied, the potential was changed to expected direction. Furthermore, no potential change was observed when solution without enzyme was supplied. These results show that the potential is changed according to the change of ratio of hexacyanoferrate (III) to (II) concentration caused by enzyme reaction. Fig. 7 (b) shows the relationship between the given and detected glucose concentrations, which shows a fairly good linearity $\left(r^{2}=0.99991\right)$.

(b)

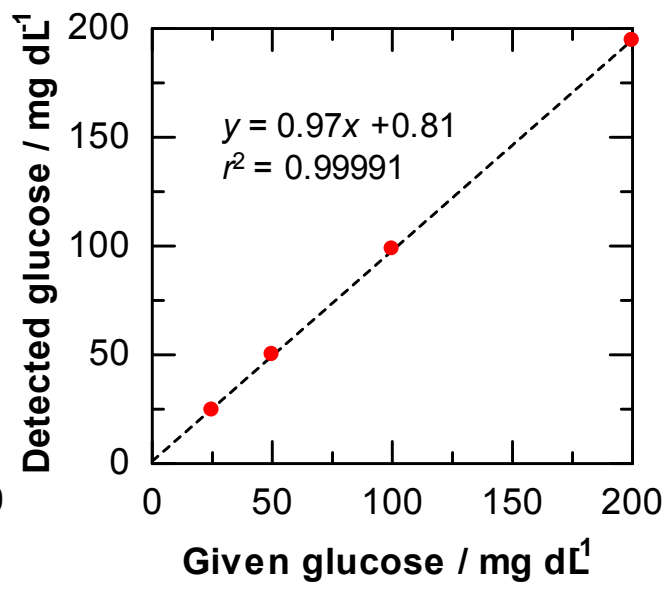

Fig. 7. (a) Time-course change of potential (median) measured for stepwise changes in glucose concentration, (b) detected glucose concentration versus given glucose concentration

\section{Conclusion}

A $32 \times 32$ array of extended-gate FET-based redox potential sensors with ferrocenylalkanethiol modified gold electrodes was developed and showed very stable detection of glucose. In the future, the 11-FUT modified FET-based sensor array will be applied to other targets, such as DNA sequencing.

\section{Acknowledgements}

This work was supported by a Grant-in-Aid for Scientific Research (S) (20226009) from the Ministry of Education, Culture, Sports, Science and Technology, Japan. The fabrication of CMOS chips was supported by ON Semiconductor Technology Japan, Ltd., and
VLSI Design and Education Center (VDEC) of the University of Tokyo, in collaboration with Synopsys and Cadence Design Systems, Inc.

\section{References}

[1] J. K. Shin, D. S. Kim, H. J. Park, G. Lim, Electroanalysis16,1912-1918 (2004); doi: 10.1002/elan.200403080

[2] C. Bian, J. Tong, J. Sun, H. Zhang, Q. xue, S. Xia, Biomed Microdev.13, 345-352 (2011); doi:10.1007/s10544

[3] Y. Ishige, M. Shimoda, M. Kamahori, Biosens. Bioelectron.24,1096-1102 doi:10.1016/j.bios.2008.06.012

(2009);

[4] K. Nakazato, Sensors 9, 8831-88 51 (2009); doi: $10.3390 / \mathrm{s} 91108831$ 